\title{
10 Qualität, Sicherheit, Transparenz: Umfassende Strategie
}

\subsection{Grundlegendes Verständnis und Rahmenkonzept}

Eine erfolgsversprechende „umfassende Qualitätsstrategie“ kann nur auf dem Hintergrund eines problemorientierten Weiterentwicklungskonzeptes des gesamten Gesundheitswesens entwickelt und umgesetzt werden. Qualität sollte nicht als defensives Instrument verstanden werden, das unerwünschte Nebeneffekte der Vergütungssystematik neutralisiert, sondern als ein integrales Bestandteil einer Zukunftsstrategie, die sich aus den zukünftigen Aufgaben einer Gesundheitsversorgung ableitet (in erster Linie Alterung und ungleiche Entwicklung der Bevölkerungsdichte). Dieses Gutachten stellt daher „fünf Ziele“ an den Anfang (s. Kap. 1.4):

- Qualitäts-statt Mengenorientierung,

- Orientierung an chronischen Mehrfacherkrankungen älterer Patienten,

- Integration und Koordination (Aufhebung der Sektorierung),

- Präventions- statt Erkrankungsbezug und

- Patienten- statt Anbieterorientierung

und entwickelt daraus einen dreidimensionalen Orientierungsrahmen (s. Abb. 49), der folgende Dimensionen umfasst: 


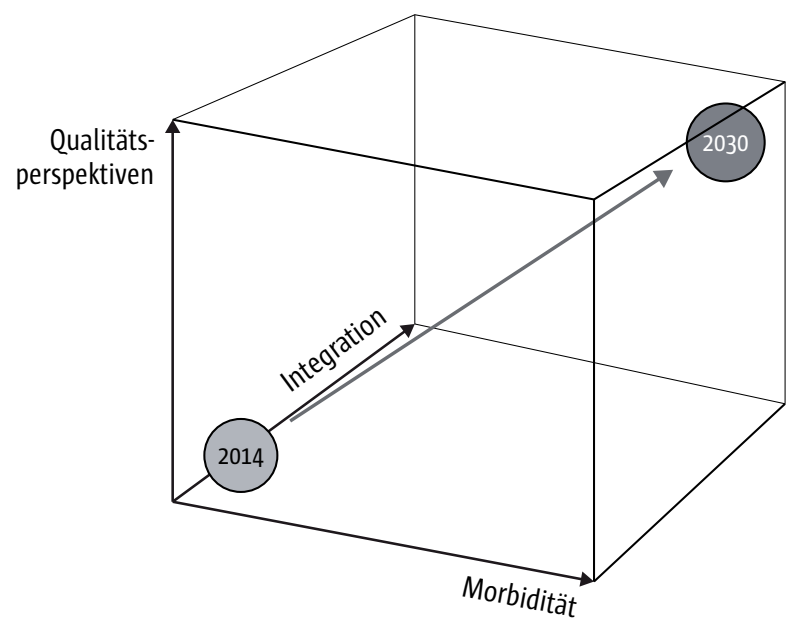

\section{Abb. 49 Dreidimensionaler Orientierungsrahmen für die Qualitätsentwicklung des Gesundheitssystems (vgl. Abb. 3, Kap. 1.4)}

1. Morbiditätsdimension, betreffend die Versorgung und Krankheitsprävention einer älter werdenden Bevölkerung mit chronischen Mehrfacherkrankungen (Chronizität/Prävention),

2. Integrationsdimension, betreffend die bessere Koordination und den Abbau der Sektorierung bei erhaltenem Zugang zur Versorgung (Struktur), und die

3. Qualitätsdimension, betreffend sechs Perspektiven, unter besonderer Beachtung der Einheit der klassischen Qualitätssicherung, des Nutzens und der Patientenorientierung (s.u.).

Dieser Orientierungsrahmen beruht auf der Einsicht, dass eine sinnvolle Diskussion über Qualität und Sicherheit nur vor dem Hintergrund einer adäquaten Abbildung der Handlungsnotwendigkeiten stattfinden kann, die durch Morbidität und Integration bzw. Struktur gegeben sind.

Um die eigentliche Qualitätsdimension besser operationalisieren zu können, werden sechs Perspektiven zugrunde gelegt, die sich aus dem Erkenntnisinteresse ableiten:

- gesellschaftliche Perspektive (Versorgung auf Populationsebene),

- Ebene des Nutzens der Behandlungsmethode(Allokation und Effizienz),

- Patientenperspektive (Selbstbestimmung, Sicherheit und Koordination),

- professionelle Perspektive (Autonomie, Garantenstellung),

- institutionelle Perspektive (Organisatorische Umsetzung),

- wissenschaftliche Perspektive (Deskription und Hypothesenbildung). 
Innerhalb dieses dreidimensionalen Orientierungsrahmens stellen Instrumente zur Qualitätssteuerung wie P4P oder Public Reporting ihrerseits komplexe Interventionen in einem überaus komplexen System dar (sog. doppelte Komplexität, s. Kap. 8.3.2), die ohne ein theoretisches Grundkonzept weder implementiert noch evaluiert werden können. Ein solches Rahmenkonzept ist in diesem Gutachten entwickelt worden (s. Kap. 9) und geht davon aus, dass Qualität und Patientensicherheit nicht (allein) als individuelle Herausforderungen zu verstehen sind, sondern als Systemeigenschaften, die zur Weiterentwicklung ihrerseits einen Systemansatz notwendig machen. Neben dem Qualitätsverständnis (s.o.) ist hierzu ein Konzept für die individuelle Verhaltensänderung notwendig; rein lerntheoretisch begründete einfache FeedbackInstrumente reichen nicht aus, stattdessen müssen Konzepte des organisatorischen Wandels und kontextbezogene Veränderungsstrategien (soziales Marketing) mit einbezogen werden. Weiterhin sind ökonomische Aspekte von großer Bedeutung, vor allem Opportunitäts- und Grenzkosten, Diskontierung, Aspekte der Risikoaversion und optimales framing müssen berücksichtigt werden, ebenso wie vergütungssystematische Konzepte, denn die Wechselwirkungen zwischen Instrumenten wie $\mathrm{P}_{4} \mathrm{P}$ und den Vergütungssystemen, in die solche Instrumente integriert werden, sind von größter Bedeutung. Letztendlich kann man auch nicht von adäquaten politischen Konzepten absehen, aktuell bietet sich das governance-Konzept an, das auf der policy-Ebene in erster Linie ein aktives direction pointing (Interpretationsebene für die anstehenden Entwicklungen), strategische Überlegungen hinsichtlich der optimalen Einsatzgebiete, die Schaffung günstiger Rahmenbedingungen und die Kontrolle eventueller negativer Nebeneffekte umfasst.

\subsection{Empfehlung an den Gesetzgeber und das Bundesministerium für Gesundheit sowie die Landesgesundheitsminister}

Gesetzgeber und Exekutive auf Bundes- und Landesebene machen die gesteigerte Bedeutung von Qualität und Patientensicherheit deutlich und legen die Grundlagen für die weitere Entwicklung fest. Sie bedienen sich dabei des direction pointing, das heißt sie geben Prioritäten und die Richtung der Entwicklung vor, ohne alle Regelungen selbst operativ umzusetzen. Wichtige Adressaten sind die Verbände, die Bundesinstitute und der Gemeinsame Bundesausschuss sowie seine beratenden Institute.

1. „Rahmenkonzept Qualitätsentwicklung“ als Grundlage: Gesetzgeber, Bundesministerium für Gesundheit und Gesundheitsministerien der Länder setzen Qualität und Patientensicherheit an die Spitze der Prioritätenliste im Gesundheitswesen. Das Bundesministerium für Gesundheit übernimmt die Aufgabe, entsprechend der internationalen Vorbilder (USA, Großbritannien) ein Rahmenkonzept zu entwickeln, das umfassend und problemorientiert die Grundlagen für die Weiterentwicklung von Qualität und Patientensicherheit 
darstellt. Das Bundesministerium ergänzt damit die Richtlinienkompetenz des Gemeinsamen Bundesausschusses nach $\mathbb{5} 92$ SGB V.

\section{Empfehlung 1:}

Das Bundesministerium für Gesundheit legt dem Gesetzgeber, den Verbänden (einschließlich Gemeinsamer Bundesausschuss) und der Öffentlichkeit ein „Rahmenkonzept Qualitätsentwicklung"vor, das ein umfassendes Qualitätsverständnis und ein Konzept zur Qualitätsentwicklung auf den Ebenen Verhaltensmodifikation, Organisationsveränderung, Ökonomie und Gesundheitssystem beinhaltet. Das Bundesministerium für Gesundheit kann die Entwicklung des Rahmenkonzeptes an den Sachverständigenrat zur Begutachtung der Entwicklung im Gesundheitswesen nach S 142 SGB V als Sondergutachten delegieren.

2. direction pointing I - Jährliches „Gutachten Qualitätsentwicklung“: Auf der Grundlage des Rahmenkonzeptes und als Zeichen der Priorität gegenüber der Öffentlichkeit, den Verbänden einschließlich dem Gemeinsamen Bundesausschuss und den Leistungsanbietern und in Ergänzung zur Qualitätsberichtserstattung nach $\mathbb{1} 137$ bzw. $\mathbb{S} 137$ a SGB V wird vom Bundesministerium für Gesundheit jährlich ein Gutachten zum Stand von Qualität und Sicherheit im deutschen Gesundheitswesen veröffentlicht. Diese Gutachten nehmen eine Gesamtsicht der Problematik ein.

\section{Empfehlung 2:}

Das Bundesministerium für Gesundheit veröffentlicht jährlich zum Jahresende ein „Gutachten Qualitätsentwicklung“ für die Bundesrepublik Deutschland. Dieses Gutachten entspricht dem Jahresgutachten des Sachverständigenrates zur Begutachtung der gesamtwirtschaftlichen Entwicklung. Das BMG kann damit (in Erweiterung der bisherigen Aufgabenstellung) den Sachverständigenrat zur Begutachtung der Entwicklung im Gesundheitswesen nach \$142 SGB V beauftragen, dessen Geschäftsstelle zu diesem Zweck personell ausgerüstet werden muss. Dem Gutachten ist ein umfassendes und problemorientiertes Qualitätsverständnis entsprechend Empfehlung 3 und 4 zugrunde zu legen; gesondert muss auf die Entwicklung im Bereich der nosokomialen Infektionen bzw. Antibiotikaresistenzen sowie auf den Bereich Patientensicherheit Stellung genommen werden.

3. direction pointing II - umfassendes Qualitätsverständnis: In dem Rahmenkonzept nach Empfehlung 1 wird verdeutlicht, dass ein adäquates Qualitätsverständnis alle Qualitätsperspektiven, wie sie im vorliegenden Gutachten formuliert worden sind (s. Kap. 1.3), berücksichtigen muss. 


\section{Empfehlung 3:}

Im Rahmenkonzept nach Empfehlung 1 wird ein umfassendes Qualitätsverständnis vertreten, das nicht nur die derzeit noch sehr lückenhafte diagnose- und prozedurenbezogene Qualitätssicherung auf eine breitere Basis stellt (insbesondere chronische und konservativ zu behandelnde Krankheitsbilder fehlen bislang vollständig, s. Kap. 2.1), sondern klarstellt, dass Aspekte der gesellschaftlichen (Populations)Perspektive, des Nutzens und Effizienz, der Patientenperspektive und weiterhin die professionelle, institutionelle und wissenschaftliche Perspektive mit berücksichtigt werden. Diese Perspektiven finden sich auch in den jährlichen „Gutachten Qualitätsentwicklung " wieder. Die wichtigsten Qualitäts- und Patientensicherheitsdefizite aus gesellschaftlicher Perspektive betreffen den Zugang zur Versorgung, die regionale Variabilität, die Mindestmengenproblematik, die Integrationsdefizite, die nosokomialen Infektionen und Antibiotikaresistenz sowie Patientensicherheit.

4. direction pointing III - problemorientiertes Qualitätsverständnis: Das Rahmenkonzept nach Empfehlung 1 basiert auf einem problemorientierten und aktiven Qualitätsverständnis. Das Rahmenkonzept muss herausstellen, dass Qualität und Qualitätssicherung nicht die Aufgabe haben, in defensiver Funktion unerwünschte Nebeneffekte eines Vergütungssystems zu neutralisieren, sondern aktiv die Entwicklung des Gesundheitssystems zu gestalten. Crundlage und unbedingte Voraussetzung jeder Qualitätsentwicklung und jedes Einsatzes von differenzierten Instrumenten zur Qualitätsverbesserung (z.B. Pay for Performance) ist daher eine eingehende Problemanalyse des Gesundheitssystems.

\section{Empfehlung 4:}

Das Rahmenkonzept nach Empfehlung 1 entwickelt das umfassende Qualitätsverständnis nach Empfehlung 3 auf der Basis einer Problemanalyse des deutschen Gesundheitswesens (vgl. "fünf Ziele" des vorliegenden Gutachtens nach Kap. 1.4). Das Rahmenkonzept stellt dabei die Qualitäts-statt Mengenorientierung, die Behandlung chronischer Mehrfacherkrankungen älterer Patienten, die Integration des Gesundheitssystems, den Präventions- und den Patientenbezug in den Vordergrund. Zukünftige Qualitätsindikatoren und -instrumente werden danach beurteilt, inwieweit sie hinsichtlich der Morbiditätsdimension (Chronizität/Prävention) und der Integrationsdimension (Überwindung der Sektorierung und Koordination) für die Weiterentwicklung des Gesundheitssystems sinnvoll erscheinen. 
5. direction pointing IV - Kohärenz der normativen Regelungen (Gesetz für Qualität und Patientensicherheit): Die gesetzlichen Regelungen zu Qualität und Patientensicherheit sind in Deutschland außerordentlich vielfältig und teilweise auch überaus detailliert, gleichzeitig fehlt es aber an Kohärenz und Priorisierung. Die Delegation der Zuständigkeit für Qualitätsfragen an den Gemeinsamen Bundesausschuss und seine beratenden Institute ist zwar sinnvoll, diese nachgeordnete Instanz ist aber aufgrund ihrer Zusammensetzung nicht in der Lage, Priorisierungen vorzunehmen und Richtungsentscheidungen zu treffen, die die mittel- und langfristige Weiterentwicklung des Gesundheitswesens reflektieren.

\section{Empfehlung 5:}

Parallel zur Entwicklung und nachfolgenden Diskussion eines „Rahmenkonzeptes Qualitätsentwicklung“ nach Empfehlung 1 wird eine Gesetzesinitiative auf den Weg gebracht, die die Kohärenz und Zielgerichtetheit der weiteren Entwicklung von Qualität und Patientensicherheit im deutschen Gesundheitswesen verbessert. Im Vordergrund steht dabei die Problemorientierung der „umfassenden Qualitätsstrategie“ und die Frage, ob die gesetzlichen Regelungen zu Qualität und Sicherheit die zukünftigen Anforderungen, denen sich unser Gesundheitssystem in Zukunft gegenübersieht (Chronizität, Multimorbidität, Alterung der Gesellschaft, Koordination, Patientenorientierung; s. Empfehlung 17 bei PR), in angemessener Form angesprochen werden.

6. Qualitätsorientierte Versorgungsplanung statt Krankenhausplanung: Die Weiterentwicklung der Krankenhausplanung in eine Versorgungsplanung ist eines der wichtigsten qualitätsbezogenen Projekte der nächsten Jahre. Die bisherige Thematik „qualitätsorientierte Krankenhausplanung“ stellt angesichts der Notwendigkeit zur Aufhebung der Sektorierung und zur vermehrten Integration der Versorgung zunehmend den falschen Zugang dar, eine Qualitätsorientierte Versorgungsplanung über alle Sektoren hinweg ist klar zu präferieren.

\section{Empfehlung 6:}

Auf Landesebene muss mittelfristig die Krankenhausplanung in eine qualitätsorientierte Versorgungsplanung, die alle Sektoren umfasst, überführt werden. Die wichtigsten Qualitätsindikatoren, von denen die Entwicklung ausgehen kann, sind der Mindestmengen- und der Zugangsindikator. Mit hoher Priorität müssen jedoch auch sog. area-Indikatoren entwickelt werden, die die Leistungsfähigkeit von populationsbezogenen, integrierten Versorgungsstrukturen beschreiben (s. Empfehlung 24). 
7. Mindestmengen als Voraussetzung für die Überführung der Krankenhaus- in die Versorgungsplanung: Allerdings kann die in Empfehlung 6 ausgesprochene Präferenz für eine qualitätsorientierte Versorgungsplanung nicht bedeuten, dass im Krankenhausbereich keine Planungsnotwendigkeit mehr besteht (ganz abgesehen von der Investitionsproblematik). Für die Bundesländer besteht die dringende Notwendigkeit, die Krankenhausversorgung in regionale integrierte Versorgungskonzepte zu überführen, um die Gesundheitsversorgung zukunftsfähig zu gestalten. Im Spannungsfeld zwischen Zentralisierung (Mindestmengen) und dezentralem Zugang (Entfernung zum Krankenhaus, Infrastrukturausstattung) besteht jedoch derzeit die Problematik, dass die Entwicklung von Mindestmengen im Gemeinsamen Bundesausschuss nicht im notwendigen Umfang vorankommt. Hinzu kommt, dass die Mindestmengen in den Zuständigkeitsbereich des Bundes und der Zugangsproblematik in denjenigen der Länder fällt, eine gute Abstimmung von Bundes- und Landesebene ist also außerordentlich wichtig.

\section{Empfehlung 7: \\ Mindestmengen als Anreiz zur Zentralisierung der Versorgung und als Counterpart der Dezentralität des Versorgungszugangs sind für die regionalen Planungskompetenzen der Länder von größter Wichtigkeit. Die Entwicklung von Mindestmengen muss u.U. unter direkter Kompe- tenz des Bundesgesetzgebers intensiviert werden (s. außerdem Emp- fehlung 8).}

8. In Deutschland werden die Potenziale zur Qualitätsverbesserung durch Mindestmengen im Krankenhausbereich nicht genutzt: Trotz des gesetzlichen Auftrags nach $\mathbb{} 137$ Abs. 3 Satz 1 Nr. 2 und Satz 2 SGB V ist die Umsetzung der Mindestmengenindikatoren durch den Gemeinsamen Bundesausschuss kaum vorangekommen, weil es zwar nicht an wissenschaftlicher Evidenz für einen grundsätzlichen Zusammenhang zwischen Leistungsmenge und Qualität fehlt, dafür aber die Grenzwerte wissenschaftlich schwer zu belegen sind. Daraus hat sich eine Kontroverse entwickelt, der die Selbstverwaltung (Gemeinsamer Bundesausschuss) nicht gewachsen ist. Nach den vorliegenden Studien sind die Qualitätsdefizite, die durch diese Blockade der Entwicklung und Umsetzung der Mindestmengen toleriert werden, von erheblichem Ausmaß, ganz abgesehen von der Behinderung einer adäquaten Fortentwicklung der Krankenhaus- und Versorgungsplanung auf Landesebene.

\section{Empfehlung 8:}

Zur Verbesserung von Qualität und Patientensicherheit ist dringend die vermehrte Entwicklung von Mindestmengen notwendig, Grenzwerte müssen nötigenfalls normativ durch den Gesetzgeber gesetzt werden 


\section{(zur Notwendigkeit von Mindestmengen in der regionalen Versorgungs- planung s. Empfehlung 7).}

9. Diskussion um den Zugang zur Versorgung beginnen: Der Zugang zur Versorgung als Gegenstück zu den Zentralisierungstendenzen ist ein wichtiges Planungskriterium für die Krankenhaus- und Gesamtversorgung. Er liegt im Zuständigkeitsbereich der Bundesländer und der dezentralen Planungseinheiten (Regionen, Kreise). Die Diskussion um diesen Qualitätsindikator muss öffentlich geführt werden. Der Indikator muss an die regionalen Besonderheiten angepasst sein und ist krankheitsspezifisch auszugestalten.

\section{Empfehlung 9:}

Eine Auseinandersetzung um den regional und krankheitsspezifisch spezifizierten Zugangsindikator muss begonnen werden, wobei der Schwerpunkt auf der Landesebene liegt. Analog anderer öffentlicher Diskurse (z.B. Energieversorgung) ist die Öffentlichkeit des Diskurses von großer Wichtigkeit. Zum Kenntnis- und Diskussionsstand in anderen Gesundheitssystemen s. Empfehlung 19 an den Gemeinsamen Bundesausschuss.

10. Nosokomiale Infektionen und Antibiotikaresistenzen stellen eines der größten Gesundheitsprobleme in Deutschland dar - die Aktivitäten zur Eindämmung dieses Problems müssen vervielfältigt werden. Nosokomiale Infektionen und Antibiotikaresistenzen sind das einzige Qualitäts- und Sicherheitsproblem, das übertragbar (ansteckend) ist, deswegen sind sie als gesamtgesellschaftliche Herausforderung anzusehen. Bei diesen behandlungsbedingten Komplikationen handelt es sich nicht um Krankenhausprobleme, sondern um ,ökologische“ Probleme des gesamten Systems, und zwar auf der Ebene des einzelnen Patienten, der Leistungserbringer, des Gesundheitssystems und der Umwelt/Gesellschaft (z.B. Tiermast). Allein im Krankenhausbereich muss man in Deutschland mit zwischen 400.000 und 60o.ooo nosokomialen Infektionen pro Jahr rechnen, ein Drittel davon ist als vermeidbar einzustufen. 7.500 bis 15.000 Todesfälle pro Jahr sind die Folge, 2.500 bis 5.000 Todesfälle gelten bei Einhaltung der empfohlenen Hygienemaßnahmen als vermeidbar. Mehrere klinische und monozentrische Studien finden weitaus höhere Werte. Administrative Daten unterschätzen die Größenordnung um ca. 50\%. Es darf nicht verkannt werden, dass die zunehmende Antibiotikaresistenz ist wahrscheinlich das wichtigste Qualitäts- und Sicherheitsproblem darstellt, das derzeit das Gesundheitswesen bedroht, insbesondere da sich die Anzeichen mehren, dass sich eine „totale“ Antibiotikaresistenz gegen alle verfügbaren Antibiotika ausbreitet. Die wichtigsten Angriffspunkte sind die Vermeidung der antibiotischen Behandlung des grippalen Infekts und der unkomplizierten Bronchitis, der Stop der Fortführung der perioperativen Antibiotikaprophyla- 
xe in der Chirurgie über die Dauer des Eingriffs hinaus, die tatsächliche Einführung von Antibiotikakonsil und regelmäßiger Besprechung der mikrobiologischen Befunde (Resistenzstatistik) auf den Stationen mit hohem Antibiotikaeinsatz (bereits gesetzlich gefordert!), und eine strengere Regulation des Antibiotikaeinsatzes in der Landwirtschaft.

\section{Empfehlung 10:}

Morbidität und v.a. die vermeidbare Sterblichkeit zwingen zu einer deutlich stärkeren Aktivität. Das Bundesministerium für Gesundheit muss in dem jährlichen "Gutachten Qualitätsentwicklung" jeweils den aktualisierten Kenntnisstand zur Häufigkeit von nosokomialen Infektionen sowie Resistenzen veröffentlichen und sollte, ähnlich wie den Institutionen im Gesundheitswesen (Krankenhäusern) im Infektionsschutzgesetz auferlegt, diese Daten jeweils dezidiert analysieren und bewerten. Präventiv angelegte Maßnahmen müssen auskömmlich gefördert werden; es ist nicht verständlich und nicht hinnehmbar, dass nach sechs Jahren ausgerechnet die Förderung der Aktion Saubere Hände, die sich auf die Verbesserung der Händedesinfektion als eines der wichtigsten Präventionsmaßnahmen konzentriert, durch das Bundesministerium für Gesundheit beendet wird. Die nach Infektionsschutzgesetz verpflichtend vorzunehmenden Meldungen von Infektionen und Resistenzen sind in das "Gutachten Qualitätsentwicklung " aufzunehmen. Händedesinfektionsmittelverbrauch pro Tag und Mitarbeiter, die Infektions- und die Resistenzstatistiken ebenso wie die Ergebnisse der Bewertung dieser Statistiken und weitere zu entwickelnde Indikatoren müssen in vollem Umfang Bestandteil des jährlichen Qualitätsberichtes nach $₫ 137$ SGB V werden. Bei der Entwicklung von Indikatoren sind die Handlungsbereiche antibiotische Behandlung des grippalen Infekts und unkomplizierten Bronchitis, Fortführung der perioperativen Antibiotikaprophylaxe und Durchführung eines Antibiotikakonsils in den Mittelpunkt zu stellen. In der Landwirtschaft ist eine Entkopplung von Antibiotikavertrieb und tierärztlicher Betreuung vorzunehmen. Es ist zu prüfen, ob die beiden mit der Thematik befassten Kommissionen im Robert Koch-Institut nach $\$ 23$ Infektionsschutzgesetz (Kommission für Krankenhaushygiene und Infektionsprävention und Kommission Antiinfektiva, Resistenz und Therapie) zur Verbesserung der Handlungsfähigkeit zusammengefasst oder durch eine Task Force gebündelt werden.

11. Patientensicherheit - Prävention stärken: Patientensicherheit ist ein normativer Begriff (Abwesenheit unerwünschter Ereignisse) und daher ein sehr direkt erfahrbarer Bestandteil der Qualitätsdiskussion. Die assoziierte Morbidität und Mortalität sind hoch: Studien und Systematische Reviews u.a. in den USA; Großbritannien und auch Deutschland zeigen, dass zwischen 
5 und 10\% der Patienten im Krankenhaus ein unerwünschtes Ereignis erleiden (zwischen 2 und 4\% ein vermeidbar, also auf Fehler zurückgehend), die die vermeidbare Sterblichkeit beträgt mindestens o,1\% (19.0oo Todesfälle pro Jahr in Deutschland). Die wichtigsten Erfolge zum Thema Patientensicherheit beruhen auf dem analytischen Zugang zu katastrophalen Einzelereignissen über die Prozessanalyse und der Betonung sog. generierender Verfahren (z.B. CIRS), die „blinden Flecken“ der Analyse zugänglich machen (s. Kap. 2.2). Erfolgreich sind Präventionsmaßnahmen entwickelt worden, die jedoch implementiert und evaluiert werden müssen, außerdem ist eine Ausweitung auf weitere Sicherheitsrelevante Bereiche notwendig. Die Aktivitäten sind bislang vom Aktionsbündnis Patientensicherheit getragen worden, das mit Unterstützung des Bundesministeriums für Gesundheit gegründet wurde und arbeiten konnte. Die genannten zukünftigen Aufgaben können jedoch in absehbarer Weise nicht allein von einem ideell arbeitenden Netzwerk getragen werden. Das Thema Patientensicherheit erscheint z.B. im Vergleich zum Grad der institutionellen Absicherung des Themas nosokomialer Infektionen (u.a. Kommissionen im Robert Koch-Institut) noch deutlich underpowered. Besonders das Thema Patientensicherheit macht auch weiterhin die Sichtbarkeit der politischen Ebene im Sinne des direction pointing notwendig.

\section{Empfehlung 11:}

Wegen der Größe des Problems und der Wichtigkeit der Thematik für die zukünftige Entwicklung des Gesundheitssystems sollte Patientensicherheit als ständiges Arbeitsgebiet des neuen Institutes für Qualitätssicherung und Transparenz im Gesundheitswesen (IQTiG) etabliert werden. In Kooperation mit dem Aktionsbündnis Patientensicherheit, das im Beirat des Institutes beteiligt sein sollte, sind insbesondere Analysen bestehender internationaler Erfahrungen und Evaluationsstudien von Präventionsmaßnahmen in Deutschland durchzuführen. Eine Delegation an wissenschaftliche Institutionen ist möglich und erwünscht (Versorgungsforschung). Eine direkte Beauftragung des Institutes durch das Bundesministerium für Gesundheit nach $\$ 137 a$ Abs. 4. Satz 2 oder 3 in der Fassung des GKV-FQWG vom 5.6.2014 ist sinnvoll.

12. Public Reporting und $\mathrm{P}_{4} \mathrm{P}$ - differenzierter Einsatz der Instrumente zur Qualitätsverbesserung: Die Analyse der internationalen Erfahrungen (s. Kap. 5 u. 6) mit den Instrumenten Public Reporting und Pay for Performance erbringt, dass beide nicht nur als komplexe Interventionen zu werten sind, die schwer zu implementieren und zu evaluieren sind, sondern dass sie keine one fits all-Lösungen darstellen. Die wichtigsten Determinanten sind Konkurrenzdruck, Möglichkeit zur Risikoselektion und Mengenorientierung. So hat public reporting seine größte Wirksamkeit in hochkompetitiven Regionen, während es in Regionen mit Monopolstruktur kaum eine Wirkung aufweist, während 
P4P vor allem dort seine Wirkung zeigt. Bei Erkrankungen, bei denen Risikoselektion einfach möglich ist (z.B. elektive operative Medizin), wird eine Wirkung beider Instrumente dadurch in Teilen konterkariert. $\mathrm{P}_{4} \mathrm{P}$ ist wenig wirksam bei Diagnosen bzw. Prozeduren mit der Option der Mengenausweitung, weil hier hohe Opportunitätskosten und niedrige Grenzkosten zur Alternative der Fallzahlsteigerung führen. Von einem undifferenzierten gemeinsamen und vor allem konsekutiven Einsatz (dual use) ist abzuraten.

\section{Empfehlung 12:}

Die Instrumente Public Reporting und Pay for Performance sind nach Problemanalyse (s. Empfehlung 4) gezielt und differenziert einzusetzen. Ein breit angelegter dual use ist abzulehnen (s. weitere Empfehlung 46).

13. Implementierungsforschung intensivieren: Die komplexen Instrumente Public Reporting und Pay for Performance müssen im komplexen System des Gesundheitswesens implementiert werden. Die Implementierungsforschung hat sich bislang vor allem mit der individuellen Verhaltensänderung (Lerntheorie, soziale Wahrnehmung) beschäftigt, war aber nicht in der Lage, tragfähige Konzepte zu entwickeln. Der Fokus muss daher erweitert werden, und zwar auf die institutionellen Grundlagen, Komplexitätstheorie, weitergehende Konzepte der Verhaltensmodifikation, ökonomische Grundlagen und politikwissenschaftliche Aspekte. Außerdem erscheint es unerlässlich, genauere Angaben zu den ökonomischen Konsequenzen der Qualitäts- und Sicherheitsdefizite zu erhalten (s. Kap. 3.8).

\section{Empfehlung 13:}

Die Kenntnisse zur Implementierung der Instrumente, die zur Verbesserung von Qualität und Patientensicherung eingesetzt werden, sind angesichts der Tragweite der Probleme zu gering. Die Implementierungsforschung muss daher gestärkt und im Fokus deutlich erweitert werden. Insbesondere müssen die organisationstheoretischen Konzepte zur professional bureaucracy (Spezialistenbürokratie, Expertenorganisation) weiter ausgearbeitet werden, es bedarf einer Vertiefung des Begriffs Komplexität im Hinblick auf die Umsetzung von qualitätsverbessernden Maßnahmen, die ökonomischen Grundlagen (principal agent) und die Wechselwirkung der Instrumente mit dem Vergütungssystem müssen erweitert werden, und auch die politologischen Konzepte in ihrer Anwendung auf das Gesundheitssystem sollten Gegenstand der Forschungsansätze sein. Weiterhin müssen die gesundheitsökonomischen Instrumente zur Abschätzung der Kosten, die durch die Qualitäts- und Sicherheitsdefizite entstehen, aktualisiert werden, damit eine zeitnahe Berichterstattung zu diesem Aspekt aufgenommen werden kann. Eine 
Finanzierung dieser Projekte im Rahmen des Innovationsfonds (Versorgungsforschung) ist anzustreben.

14. Direction pointing V - Einbeziehung der Zivilgesellschaft: Das Thema Qualität und Patientensicherheit ist von großer Bedeutung. Konzepte des sozialen Marketings verweisen zur Lösung solcher übergeordneten Problemstellungen auf die große Bedeutung des Kontextes hin, in dem die Probleme diskutiert und Lösungsmöglichkeiten angeboten werden. Ganz entscheidend ist dabei die Öffentlichkeit, vor allem soweit sie nicht direkt in die Problemstellung involviert ist. Wie vor einigen Jahren mit der Initiierung der Aktion Saubere Hände praktiziert, sollte auch - angesichts des Problemdrucks - diskutiert werden, wie man die „Zivilgesellschaft“, d.h. die Teile der Öffentlichkeit, die nicht direkt im Gesundheitswesen tätig oder direkt wirtschaftlich daran interessiert sind, beteiligt. Die Begründung liegt auf der Hand, denn die Krankheitskosten werden ja in hohem Maße gesellschaftlich getragen. Es gibt internationale Vorbilder, so z.B. die Leapfrog-Initiative in den USA, die sich der Patientensicherheit widmet und aus Unternehmen besteht, die nicht direkt am Gesundheitswesen beteiligt sind (aber ihre Mitarbeiter versichern).

\section{Empfehlung 14:}

Vom Bundesministerium für Gesundheit wird ein „Beirat Qualität und Patientensicherheit" gegründet, in dem Gruppen und Verbände, die sich außerhalb des Gesundheitswesens befinden, Mitglieder entsenden (Kirchen, Gewerkschaften, Arbeitgeber etc.). Dieser Beirat berät das Bundesministerium für Gesundheit sowie die Landesgesundheitsminister sowie die Einrichtungen der Selbstverwaltung. Der Beirat kann Vorschläge machen zur weiteren Entwicklung des Themas Qualität und Sicherheit.

\subsection{Empfehlungen an den Gemeinsamen Bundesausschuss und die beratenden wissenschaftlichen Institute}

Im Jahr 2005 wurde die Zuständigkeit für die Thematik Qualität vom Bundesministerium für Gesundheit auf den GBA übertragen (s. Kap. 7.2.2). Gerade im Zusammenhang mit dem neuen Institut für Qualitätssicherung und Transparenz im Gesundheitswesen ( $\$$ 137a SGBV [neu], IQTiC) ist zu hoffen, dass durch die Öffentlichkeit, die dort der Entscheidungsfindung zur Fragen der Qualität und Sicherheit zukommt, eine kohärente Vorgehensweise eingeschlagen wird. Das unterstützende Umgehen des CBA mit der Thematik Patientensicherheit Anfang in den letzten Jahren war diesbezüglich sehr ermutigend.

1. Dualität IQWiG/IQTiG kritisch hinterfragen und optimale Koordination gewährleisten: Durch die Schaffung des Institutes für Qualitätssicherung 
und Transparenz im Gesundheitswesen (IQTiG) nach $\$ 137$ a SGB V [neu] in der Fassung des FQWG vom 5.6.2014 ist es zu einer Dualität zweier wissenschaftlicher, den GBA beratenden Institute gekommen, die beide schon im Namen den Begriff „Qualität“ bzw. „Qualitätssicherung“ tragen. Nach der Beauftragung in $\$ 137 \mathrm{a}$ (IQTiG) bzw. $\$ 139 \mathrm{ff}$. (IQWiG) SGB V decken beide Institute wichtige Qualitätsperspektiven ab (s. Kap. 1.3 und 7.2.2), und zwar die vergleichende Qualitätssicherung im Sinne der diagnose- und prozedurenbezogenen Qualitätssicherung (IQTiG) und die Nutzen- bzw. Allokationsperspektive (IQWiG). Grundsätzlich muss festgehalten werden, dass dies einem sinnvollen, einem umfassenden Qualitätsverständnis folgenden Herangehen an die Thematik Qualität und Patientensicherheit (s. Empfehlung 3) nicht zuträglich ist bzw. Risiken in sich birgt (widersprüchliches Grundverständnis, gegeneinander Ausspielen durch Dritte, widersprüchliches methodisches Herangehen etc.).

\section{Empfehlung 15:}

Der GBA ist sich der Dualität der Institute IQTiG und IQWiG hinsichtlich des Themas Qualität und Patientensicherheit bewusst und berücksichtigt in den Geschäftsordnungen beider Institute sowie in deren Beauftragung die Tatsache, dass sowohl die diagnose- und prozedurenbezogene Qualitätssicherung als auch die Nutzen- und Allokationsperspektive wichtige Qualitätsperspektiven darstellen und nicht gegeneinander aufzurechnen sind. Der GBA wirkt darauf hin, dass die Methodenpapiere beider Institute optimal aufeinander abgestimmt sind.

2. Interessenskollision bei der Auftragsvergabe an das IQTiG vermeiden: Die „parteiischen Mitglieder“ (Deutsche Krankenhausgesellschaft, Kassenärztliche Bundesvereinigungen und die Krankenkassen sind typischerweise selbst Gegenstand einer Qualitätssicherung, die ihren Namen verdient: es werden Qualitätsdefizite aufgedeckt, die zur Änderung des Verhaltens und der Geschäftspolitik führen. Da diese Mitglieder des GBA allerdings nach $\$ 137 \mathrm{a}$ Abs. 4 [neu] die Möglichkeit haben, das IQTiC zu beauftragen, wäre die Unabhängigkeit des Institutes nicht mehr gegeben, sofern sie auch im Vorstand vertreten sein sollten. Die Situation wäre einer (hypothetischen) Situation gleichzusetzen, in der die pharmazeutischen Unternehmen im Vorstand des IQWiG über die personelle Besetzung des Institutsleiters entscheiden und die Auftragsvergabe mit zu bestimmen hätten.

\section{Empfehlung 16:}

Die Unabhängigkeit des IQTiG ist zu gewährleisten. Im Vorstand des IQTiG dürfen ausschließlich die unparteiischen Mitglieder des GBA vertreten sein. 
3. Die bisher angewandten Indikatoren nach $\mathbb{S}$ 137/137a SGB V auf Problemorientierung überprüfen und Handlungsbedarf darlegen: Das nationale „Rahmenkonzept Qualitätsentwicklung“ wird problemorientiert erstellt und nimmt insbesondere die durch Alterung der Gesellschaft, Chronizität und Multimorbidität der Erkrankungen sowie regional ausgeprägte Abnahme der Bevölkerung entstehenden Gesundheitsprobleme in den Fokus (s. Empfehlung 4). Das vorliegende Gutachten kommt zu dem Schluss, dass selbst die auf die stationäre Gesundheitsversorgung beschränkte diagnose- und prozedurenbezogene Qualitätssicherung nach $\$ 137$ a SGB V diesen Versorgungssektor nur sehr unvollständig abgedeckt und daher einer energischen Neuorientierung bedarf (s. Kap. 2.1). Es wird weiterhin als möglich dargestellt, dass Public Reporting (ebenso wie $\mathrm{P}_{4} \mathrm{P}$ ) bei ausschließlichem Einsatz im Bereich der Akutmedizin den Mengenanreiz des DRG-Systems noch verstärkt, indem es die Aufmerksamkeit auf diese Erkrankungen lenkt und dadurch zu einer weiteren Vernachlässigung chronischer, konservativ zu behandelnder Erkrankungen und der Prävention führt.

\section{Empfehlung 17:}

Der GBA beauftragt das IQTiG, eine Analyse der bislang nach $\$ 137 / 137 a$ SGB V gebräuchlichen Indikatoren hinsichtlich ihrer Eignung vorzunehmen, die genannten Probleme der alternden Gesellschaft zu adressieren. Die Analyse soll beispielhaft auch internationale Beispiele umfassen und darlegen, ob und inwiefern die Problemorientierung dort umgesetzt wurde. Dabei soll auch der Frage nachgegangen werden, ob es Anhaltspunkte dafür gibt, dass Public Reporting den Mengenanreiz eines DRGSystems verstärkt. Auf dieser Grundlage sollen Vorschläge gemacht werden, wie die Problemorientierung der Indikatoren in Deutschland verbessert werden kann.

4. Die bisher angewandten Indikatoren nach $\$ 137 / 137$ a SGB V auf die Frage hin untersuchen, welche Qualitätsperspektiven von ihnen angesprochen werden, und Handlungsbedarf darlegen: Das nationale „Rahmenkonzept Qualitätsentwicklung“ (s. Empfehlung 1) geht von einem umfassenden Qualitätsverständnis aus, das über die diagnosen- und prozedurenbezogene Qualitätssicherung hinaus die gesellschaftliche Perspektive (Versorgung auf Ebene der Population), die Perspektive des Nutzens und der Allokation, der Patienten, der Professionen, der Institutionen und der Wissenschaft einnimmt (s. Empfehlung 3).

\section{Empfehlung 18:}

Der GBA beauftragt das IQTiG, eine Analyse der bislang nach \$137/137a SGB V gebräuchlichen Indikatoren hinsichtlich ihrer Eignung vorzuneh- 
men, die genannten sechs Qualitätsperspektiven abzudecken. Die Analyse soll beispielhaft auch internationale Beispiele umfassen und darlegen, ob und inwiefern dort unterschiedliche Qualitätsperspektiven beschrieben werden. Auf dieser Grundlage sollen Vorschläge gemacht werden, wie die Indikatoren in Deutschland weiterentwickelt werden müssen, um zu einer umfassenden Beschreibung der unterschiedlichen Qualitätsperspektiven zu kommen.

5. Der Indikator „Zugang zur Versorgung“ hat eine geographische, eine soziale, eine Angebots- und eine Patientendimension. In Deutschland erscheint die Zugangsproblematik im Vergleich zu anderen Ländern zunächst als gering ausgeprägt. Allerdings wird der Zugangsindikator in Zukunft als Gegenstück zu den Zentralisierungstendenzen z.B. durch die Mindestmengen (s. Empfehlung 7) eine wichtige Rolle in der regionalen Versorgungsplanung der Bundesländer spielen (s. Empfehlung 9). Bereits jetzt sind Zugangsprobleme in Deutschland nicht völlig zu übersehen, so geben in internationalen Vergleichsuntersuchungen 2o bis 30\% der Patienten an, sie hätten Wartezeiten auf einen Arztkontakt von mehr als 5 Tagen, speziell bei Facharztterminen, und der Zugang sei auch aus finanziellen Cründen erschwert. Bei GKV-Versicherten zeigt sich gegenüber Privatversicherten eine Verlängerung der Wartezeit auf einen Facharzttermin um den Faktor 3 (s. Kap. 3.2.1).

\section{Empfehlung 19:}

Der GBA beauftragt das IQTiG, eine nach Erkrankungen und regionalen Charakteristika (z.B. Ballungsgebiete, ländliche Gebiete) gegliederte Übersicht über die international gebräuchlichen Zugangsindikatoren zu erstellen und Empfehlungen für die Umsetzung in Deutschland speziell zur Verwendung in der Versorgungsplanung der Länder und Regionen zu erarbeiten. Fragen der Verkehrsinfrastruktur sind einzuschließen.

6. Qualitätssicherung nach $\mathbb{S} 137$ : Indikatoren und Parameter zur quantitativen Erfassung unterscheiden. Von zentraler Bedeutung für die heutige Qualitätssicherung ist die Unterscheidung von der quantitativen Erfassung unerwünschter Ereignisse und dem Einsatz von Indikatoren. Die Validität von quantitativen Methoden bezieht sich auf die Messung des unerwünschten Ereignisses, die Validität von Indikatoren darauf, ob das gemessene Ereignis andere Ereignisse bzw. die Qualität vorhersagt. Die Infektionsepidemiologie zur Erfassung von nosokomialen Infektionen hat eine jahrzehntelange Erfahrung in der Etablierung international standardisierter Falldefinitionen, die die internationale Kooperation und den Vergleich über Institutionen und Länder hinweg möglich machen. Indikatoren bieten hingegen ein Monitoring von Versorgungsbereichen, sind aber weniger zum Vergleich von Institutionen 
geeignet. Epidemiologische Falldefinitionen (sog. quantitative Verfahren, s. Kap. 2.3) müssen eine möglichst optimale Balance zwischen Sensitivität und Spezifität aufweisen, während bei Indikatoren ganz die Sensitivität im Vordergrund steht. Die ,intrinsische Ungerechtigkeit“von Indikatoren ist der Tatsache geschuldet, dass aus statistischen Gründen eine hohe Sensitivität immer zu Lasten der Spezifität geht, ein sensitiver Indikator folglich immer einige solcher Einrichtungen als „auffällig“ identifiziert, die gar kein Qualitätsproblem haben (s. Kap. 2.4.2).

\section{Empfehlung 20:}

Der GBA gibt dem IQTiG den vordringlichen Auftrag, das Konzept der "Qualitätsmessung" nach $§ 137$ SGB V zu aktualisieren und in ein Konzept zu überführen, das die differentielle Verwendung von quantitativen Qualitätsparametern und Qualitätsindikatoren zum Gegenstand hat (generierende Verfahren wie z.B. CIRS bleiben unberührt). Quantitative Parameter (z.B. Mortalität, nosokomiale Infektionen etc.) liefern entscheidende Hinweise auf Problemstellungen in unserem Gesundheitssystem und in einzelnen Institutionen, Indikatoren dienen dagegen dem Monitoring und geben Hinweise auf das Risiko ungünstiger Entwicklungen. Das Ziel dieses Auftrages an das IQTiG sollte es sein, dass Indikatoren in einer Form verwendet werden, dass die Institutionen z.B. nachzuweisen haben, inwieweit sie eine Analyse des dem Monitoring unterworfenen Leistungsbereiches durchgeführt haben. Quantitative Auffälligkeiten sollten Anlass dafür sein, dass Institutionen z.B. Maßnahmen nachzuweisen haben, die eine Wiederholung unwahrscheinlich machen.

7. Prozessindikatoren müssen gegenüber Ergebnisindikatoren wieder gestärkt werden. Für den Einsatz von Indikatoren speziell im Rahmen eines public reporting Einsatzes sind Ergebnisindikatoren wegen der Notwendigkeit zur Risikoadjustierung und der Benachteiligung kleiner Einrichtungen bei Diagnosen mit geringer Fallzahl kritisch zu sehen, außerdem fördern die „bad apples“ nicht die Motivation, da wegen der ex-post Perspektive das „Kind schon im Brunnen " ist. Prozessindikatoren müssen (meist) nicht risikoadjustiert werden und entsprechen dem präventiven Charakter, den man im Qualitäts- und Risikomanagement im Allgemeinen einnehmen möchte. Hinzu kommt, dass die Verantwortlichkeiten klar sind: anders als bei Ergebnisindikatoren, wo die Zurechenbarkeit in der Behandlungskette oft umstritten ist, ist bei Prozessindikatoren die Zuordnung eindeutig. International haben praktisch alle umfangreichen Qualitätsprojekte mit Prozessindikatoren begonnen, wobei Komplikationen wie nosokomiale Infektionen auch als Prozessindikatoren verstanden werden (s. Tab. 2 in Kap. 2.4.5). Neben diesen grundsätzlichen Überlegungen ist jedoch noch entscheidender, dass die in Zukunft notwendige Ausrichtung des Gesundheitswesens auf die Behandlung von chronischen 
Mehrfacherkrankungen aus dem konservativ zu behandelnden Formenkreis und die stärkere Betonung der Koordination der Behandlung (statt die Ausrichtung auf die Behandlung von Akuterkrankungen) die Behandlungsprozesse viel wichtiger erscheinen lassen.

\section{Empfehlung 21:}

Prozessindikatoren entsprechen den zukünftigen Herausforderungen des Gesundheitswesens besser als Ergebnisindikatoren, sind gut zuzuordnen und bedürfen keiner Risikoadjustierung, sie sind daher auch in Deutschland wieder vermehrt in den Vordergrund zu rücken (z.B. in der Behandlung von chronischen Erkrankungen, Koordination bei Mehrfacherkrankungen, regionale Koordination). Für die weitere Entwicklung ist der Einsatz von Ergebnisindikatoren nicht ausgeschlossen, wenn deren methodische Probleme erfolgreich bearbeitet wurden.

8. Routinedaten mit Vorsicht nutzen - sie übersehen wegen ihrer mangelnden Sensitivität einen Großteil der Ereignisse. Routinedaten ( $\$ 21 \mathrm{KHEntgG-}$ es, $\mathbb{} 299$ Abs. 1 SGB V, $\mathbb{S} 301$ SGB V, $\mathbb{S}$ 303e Abs. 1) sind vorhanden und sollten daher differenziert, insbesondere zur Validierung von klinischen Daten und zur Analyse von klärungsbedürftigen Sachverhalten (z.B. Dekubitus) eingesetzt werden (s. Kap. 2.4.6). Die Sensitivität ist aber sehr niedrig, daher ist die wichtigste Eigenschaft, die einen guten Indikator auszeichnet, nicht erfüllt. $\mathrm{Zu}$ warnen ist vor der Annahme, die Reliabilität von Routinedaten sei per se hoch; vergütungsrelevante Daten sind immer unter dem Blickwinkel der ökonomischen Anreizbildung zu betrachten. Deswegen wird eine aussagekräftige Qualitätssicherung nicht überwiegend oder gar allein auf der Basis von Routinedaten aufzubauen sein.

\section{Empfehlung 22:}

Administrative (Routine-, Sozial-)Daten sind immer primär unter dem Blickwinkel des ökonomischen Anreizes zu interpretieren und wegen ihrer geringen Sensitivität zwar für Sonderfragestellungen interessant, aber als Basis für eine Qualitätssicherung nicht zu verwenden.

9. Die transsektorale Qualitätssicherung kann die sektorale Perspektive nicht überwinden. Keiner der 31 in der externen Qualitätssicherung nach $\$$ 137a SGB V aufgenommen Krankheitsbilder bzw. Prozeduren weisen eine sektorenübergreifende Perspektive auf, das AQUA-Institut hat aber gemäß dem gesetzlichen Auftrag eine kleine Zahl von transsektoralen Indikatoren entwickelt. Da die Entwicklung sehr aufwändig ist, bleibt es naturgemäß bei einzelnen Diagnosen, für die eine transsektorale Qualitätssicherung umgesetzt werden kann („transsektorale Tunnel“). Die Sektorlogik wird daher nicht 
aufgehoben, es ergibt sich kein integriertes Bild für die Versorgung einer Population (s. Kap. 2.4.8). Chronizität und Multimorbidität können nicht sinnvoll beschrieben werden, die transsektorale Qualitätssicherung hat für die Gestaltung des zukünftigen Gesundheitswesens keine Perspektive. Interessant sind die routinedatenbasierten Projekte des WIdO-Institutes, die stationäre und ambulante Daten koppeln, aber hier kommt ebenfalls nur ein Bild für einzelne Diagnosen zustande, und außerdem kann es nicht zu einem sinnvollen Feedback kommen, denn den Krankenhäusern obliegt nicht die Verantwortung für die poststationäre ambulante oder rehabilitative Behandlung. Die Krankenhäuser können weiterhin nicht auf die deanonymisierten Daten zugreifen und die Fälle daher nicht analysieren, so dass kein Lerneffekt zustande kommt.

\section{Empfehlung 23:}

Die sog. transsektorale diagnosen- oder prozedurenbezogene Qualitätssicherung sollte zugunsten eines regionalen Verständnisses von Indikatoren (area-Indikatoren, s.u.) verlassen werden. Abgesehen von der Aussichtslosigkeit, mit einer diagnosenbezogenen Betrachtungsweise zu einer sinnvollen Abbildung des Gesundheitssystems in seiner zukünftigen Ausprägung zu gelangen, überwindet sie nicht die sektorale Perspektive, sondern läuft Gefahr, die Sektorierung des Gesundheitswesens noch zu verstärken.

10. Area-Indikatoren beschreiben die Versorgung von Populationen und müssen wegen der fortschreitenden Integration rechtzeitig entwickelt werden. Die Alternative zur sektoralen Perspektive ist nicht die transsektorale Perspektive, sondern die Versorgung von Populationen. Die Entwicklung in Deutschland kann sich auf eine relativ große Anzahl von Versorgungsformen stützen (zuletzt ambulante spezialärztliche Versorgung), so dass die Tendenz zur Integration langsam aber stetig zunimmt. Die Qualitätsdiskussion muss diese Entwicklungen antizipieren, damit rechtzeitig Indikatoren vorliegen, die die unerwünschten Effekte hochgradig integrierter Konzepte beschreiben können. Es ist auch die Übernahme der Versicherungsfunktion durch die kooperierenden Leistungserbringer mit einzubeziehen (Aufhebung des providerpayer splits in Managed Care), die zwar eine wünschenswerte Tiefe und Breite der Integration gewährleistet, aber auch Nebeneffekte wie Vorenthaltung von Leistungen mit sich bringen kann.

\section{Empfehlung 24:}

Der Gemeinsame Bundesausschuss beauftragt das IQTiG mit hoher Dringlichkeit, die Entwicklung von area-Indikatoren voranzutreiben, die die regionale Versorgung beschreiben. Im ersten Schritt sollte eine Bestands- 
aufnahme der international gebräuchlichen Indikatoren dieser Art vorgenommen werden, in zweiten Schritt die bereits in Deutschland verwendeten Indikatoren zusammengestellt werden. In Abstimmung mit der regionalen Versorgungsplanung auf der Ebene der Bundesländer, dem Mindestmengen- und dem Zugangsindikator (s. Empfehlungen 7-9) müssen die Indikatoren insbesondere die Anforderungen der zukünftigen Gesundheitsversorgung in den Fokus nehmen (Koordination, Behandlungsprozesse etc.).

11. Auch in Deutschland gibt es eine medical practice variation je nach Diagnose in einer Bandbreite um den Faktor 2 bis 4. Die Zahl der Hüft-OPs schwankt um den Faktor 2,6, die der Hysterektomien um den Faktor 4,4 und die Zahl der Eingriffe in der Wirbelsäulenchirurgie um den Faktor 4, 8. Im Bereich der bradykarden Herzrhythmusstörungen (Auswahl aus dem AQUA-Qualitätsbericht) schwanken nach den Angaben des AQUA-Qualitätsberichtes die Indikatoren bzw. Messparameter um den Faktor 1, 9 bis 4 (s. Kap. 3.2.2). Diese kleinräumige Unterschiede in der Versorgungsdichte und -qualität entsprechen genau dem räumlichen Horizont, unter dem die Entwicklung von areaIndikatoren (s. Empfehlung 24) vorgenommen werden muss und steht daher in engem Zusammenhang mit der Planung und Qualitätsbeschreibung der regionalen integrierten Versorgungsstrukturen.

\section{Empfehlung 25:}

Im engen Zusammenhang mit der Thematik der area-Indikatoren muss die kleinräumige Varianz der Versorgung in Deutschland genauer untersucht werden. Der Gemeinsame Bundesausschuss beauftragt das IQTiG daher, die Varianz der Versorgung parallel zur Entwicklung von area-Indikatoren anzugehen.

12. Indikatoren auf der Basis von Patientenerfahrungen und Patient-Reported Outcome Measures sind international gebräuchlich und bieten valide und relevante Informationen über die Qualität der Gesundheitsversorgung. Patientenvertreter spielen mittlerweile eine wichtige Rolle im deutschen Gesundheitswesen, als Akteure im sog. Qualitätswettbewerb und als Vertreter in den Gremien des Gesundheitswesens. Patientenerfahrungen und PatientReported Outcome Measures betreffen vornehmlich den Bereich der Prozessindikatoren (Koordination, Information, Kommunikation), wo gerade aus der Patientenperspektive weiterhin gravierende Mängel feststellbar sind. Bei Entlassung aus dem Krankenhaus haben ein Viertel der Patienten keine Instruktionen, für bis zur Hälfte der Patienten wurden keinerlei Arrangements getroffen. Befunde fehlen bei 10\% der Patienten, zwischen $32 \%$ bzw. 17\% der Patienten beobachten, dass Fachärzten keine Anamnese der Hausärzte vorlag 
bzw. der Hausarzt keinen Bericht vom Facharzt erhalten hat. Es ist besonders der Übergang zwischen den verschiedenen Institutionen und Versorgungsektoren, der für das Auftreten von unerwünschten Ereignissen besonders relevant ist. In einer monozentrischen holländischen Studie erlitten in den ersten vier Wochen nach Entlassung aus dem Krankenhaus 25\% der Patienten ein unerwünschtes Ereignis. Die regelmäßige Überprüfung der Medikation (sog. medication reconciliation) ist immer noch nicht umgesetzt (s. Kap. 2.4.7 und 3.4). Die laut $\$ 137 \mathrm{a}$ [neu] SGB V in der Fassung des FQWG vom 5.6.2014 als Aufgabe für das IQTiC formulierte Einbeziehung von Patientenerfahrungen in die Entwicklung von Qualitätsindikatoren stellt daher eine sehr bedeutsame Entwicklung dar.

\section{Empfehlung 26:}

Der Gemeinsame Bundesausschuss betont in seiner Auftragsgestaltung an das IQTiG die Einbeziehung von Patientenerfahrungen in die Entwicklung von Qualitätsindikatoren und nimmt speziell die von Patienten geforderte Prozess- und Koordinationssicht zum Anlass, die notwendige Schwerpunktverschiebung von Ergebnis- zu Prozessindikatoren zu beschleunigen (s. Empfehlung 21).

13. Patientensicherheit wird „normales“ Risiko- und Qualitätsmanagement, aber spezielle Patientengruppen mit hohem Risiko (z.B. ältere Patienten) müssen verstärkt im Fokus bleiben. Der demographische Wandel rückt ältere Patienten mit Mehrfacherkrankungen in den Mittelpunkt, die besonders unter Koordinationsmängeln der Versorgung, unter Stürzen, unter unerwünschten Ereignissen im Krankenhaus und der weit verbreiteten Polypharmakotherapie leiden. Obwohl es Empfehlungen zur Vermeidung von unerwünschten Arzneimittelereignissen gibt (z.B. Priscus-Liste), erhalten rund $20 \%$ der älteren Patienten ein Medikament, das nach Priscus-Liste nicht empfohlen wird.

\section{Empfehlung 27:}

Der Gemeinsame Bundesausschuss sowie das IQTiG unterstützen die bisherigen Projekte im Rahmen des Aktionsplans Arzneimitteltherapiesicherheit und hier insbesondere die Thematik der Polypharmakotherapie chronisch und mehrfacherkrankter Patienten.

14. Patientensicherheit wird als besondere Ausprägung des Themas Qualität langfristig relevant bleiben, dies ergibt schon allein durch die quantitative Mächtigkeit des Problems (s. Kap. 3.3.3 und Empfehlung 11). 


\section{Empfehlung 28:}

Wegen der Größe des Problems und der Wichtigkeit der Thematik richtet der Gemeinsame Bundesausschuss entsprechend Empfehlung 11 Patientensicherheit als ständiges Arbeitsgebiet des neuen Institutes für Qualitätssicherung und Transparenz im Gesundheitswesen ein.

15. Durch die Choosing Wisely-Kampagne in den USA und Kanada wurde die Thematik der Überversorgung international wieder in das Zentrum der Diskussion gerückt. Überversorgung besteht bei Leistungen ohne Netto-Nutzen und ist oft mit Fehlversorgung verbunden (s. Kap. 3.3.1). Es handelt sich um Verfahren, die aus unterschiedlichen Gründen nicht evaluiert sind oder bei denen in der Evaluation kein Nutzen festgestellt werden konnte (insbesondere im Bereich der sog. IGeL-Versorgung (,individuelle Gesundheitsleistungen“), jährliches Volumen von 1 bis 1,5 Mrd. €), mangelhaft evaluierte medizintechnische Verfahren (eine Zulassung analog der Arzneimittel auf der Basis eines Nutzennachweises ist hier bislang nicht vorgesehen), die Indikationsausweitung von Verfahren ohne zusätzlichen Nutzen (z.B. diagnostische Herzkatheteruntersuchungen und DRG-getriebene Mengenausweitungen), die Anwendung von Verfahren, bei denen die Studienergebnisse nicht vollständig publiziert wurden (als besonders drastisches Beispiel sind hier Oseltamivir (Tamiflu) und Zanamivir (Relenza) gegen Influenza zu nennen) und die Verschreibung von Medikamenten, die entweder als reine Me Too-Präparate (Nachahmer-Präparate) einzuschätzen sind oder bei denen es bewährte Generika gibt (Einsparpotenzial für die GKV pro Jahr bis zu 2 Mrd. €).

\section{Empfehlung 29:}

Der Gemeinsame Bundesausschuss beauftragt das IQWiG, die Optionen zur Gründung einer deutschen Choosing Wisely-Kampagne zu prüfen und diese zu unterstützen. Der Gemeinsame Bundesausschuss beauftragt das IQWiG mit der Erstellung einer zusammenfassenden Übersicht über die im deutschen Gesundheitssystem vorhandenen Überversorgung und einer Abschätzung der dadurch entstehenden Qualitätsdefizite (Fehlversorgung) und jährlichen Kosten.

16. Entgegen der allgemeinen Ansicht gibt es im deutschen Gesundheitswesen eine Unterversorgung bzgl. zahlreicher Behandlungsmethoden. Unter Unterversorgung versteht man die Vorenthaltung einer Behandlungsmethode mit nachgewiesenem Nutzen (3.3.2.). Zu einigen Aspekten ist schon unter „Zugang zur Versorgung“ Stellung genommen worden (s. Empfehlung 9, s. Kap. 3.2.1). Weitere Themen betreffen die Händedesinfektion oder eine adäquate Schmerztherapie. Durch mangelnde Händedesinfektion, seit 
dem Jahr 1847 wissenschaftlich evaluiert, versterben jährlich in Deutschland 2.500 bis 5.000 Patienten. Bei einer Befragung von Patienten in sieben westlichen Ländern gaben in einer Studie des Commonwealth Fund 18\% der Patienten aus Deutschland an, sie hätten im Krankenhaus keine oder keine genügende Schmerztherapie erhalten. In einer Studie der Initiative „Schmerzfreies Krankenhaus" gaben 29,5\% der operierten und 36,8\% der konservativ behandelten Patienten an, in Ruhe moderate bis starke Schmerzen zu haben, 15 und 39\% der Patienten erhielten trotz Schmerzen keine Schmer. Ein weiteres Thema, das im Auge behalten werden sollte, ist eine mögliche Unterversorgung durch eine Versicherungskrise (malpractice crisis), so wie sie zuletzt bei der Hebammenversorgung diskutiert wurde. Es ist durchaus möglich, dass in Zukunft Krankenhäuser (und andere Einrichtungen) Leistungen nicht mehr anbieten können, weil sie keine Haftpflichtversicherung erhalten.

\section{Empfehlung 30:}

Der Gemeinsame Bundesausschuss beauftragt das IQWiG mit der Erstellung einer zusammenfassenden Übersicht über die im deutschen Gesundheitssystem vorhandenen Unterversorgung und einer Abschätzung der dadurch entstehenden Qualitätsdefizite (Fehlversorgung) und jährlichen Kosten. Ein dabei speziell zu betrachtender Aspekt stellt die Versicherbarkeit der Leistungsanbieter dar, die vom IQWiG in unabhängiger Form dargestellt werden sollte. 